\title{
Trace Formulas for Schrödinger Operators in Connection with Scattering Theory for Finite- Gap Backgrounds
}

\author{
Alice Mikikits-Leitner and Gerald Teschl

\begin{abstract}
We investigate trace formulas for one-dimensional Schrödinger operators which are trace class perturbations of quasi-periodic finite-gap operators using Krein's spectral shift theory. In particular, we establish the conserved quantities for the solutions of the Korteweg-de Vries hierarchy in this class and relate them to the reflection coefficients via Abelian integrals on the underlying hyperelliptic Riemann surface.
\end{abstract}

\section{Introduction}

Trace formulas for one-dimensional (discrete and continuous) Schrödinger operators have attracted an enormous amount of interest recently (see e.g. [3], [18, [20], 27], 30], 34, 39]). However, most results are in connection with scattering theory for a constant background. On the other hand, scattering theory for one-dimensional Schrödinger operators with periodic background is a much older topic first investigated by Firsova in a series of papers 9 - 11. Nevertheless, many questions which have long been answered in the constant background case are still open in this more general setting.

The aim of the present paper is to help filling some of these gaps. To this end, we want to find the analog of the classical trace formulas in scattering theory for the case of a quasi-periodic, finite-gap background. In the case of zero background it is well-known that the transmission coefficient is the perturbation determinant in the sense of Krein [22] (see e.g., [19], 32, 38, see also [15, [16] and the references therein for generalizations to non trace class situations) and our first aim is to establish this result for the case considered here; thereby establishing the connection with Krein's spectral shift theory. Our second aim is to find

2000 Mathematics Subject Classification. Primary 34L25, 35Q53; Secondary 81U40, 37K15.

Key words and phrases. Scattering, KdV hierarchy, Trace formulas.

Work supported by the Austrian Science Fund (FWF) under Grant No. Y330.

in Spectral Theory and Analysis, J. Janas (ed.) et al., 107-124, Oper. Theory Adv. Appl. 214, Birkhäuser, Basel, 2011. 
a representation of the transmission coefficient in terms of the scattering data the analog of the classical Poisson-Jensen formula.

Moreover, scattering theory for one-dimensional Schrödinger operators is not only interesting in its own right, it also constitutes the main ingredient of the inverse scattering transform for the Korteweg-de Vries (KdV) hierarchy (see, e.g., [5], 28). Again the case of decaying solutions is classical and trace formulas for this case were studied exhaustively in the past (cf. 14 and the references therein). Here we want to investigate the case of Schwartz type perturbations of a given finitegap solution. The Cauchy problem for the KdV equation with initial conditions in this class was only solved recently by Egorova, Grunert, and Teschl [8] (see also [6, [7, [12]). Since the transmission coefficient is invariant when our Schrödinger operator evolves in time with respect to some equation of the KdV hierarchy, the corresponding trace formulas provide the conserved quantities for the $\mathrm{KdV}$ hierarchy in this setting.

Our work extends previous results for Jacobi operators by Michor and Teschl [29], 36. For trace formulas in the pure finite-gap case see Gesztesy, Ratnaseelan, and Teschl [17] and Gesztesy and Holden [13.

\section{Notation}

We assume that the reader is familiar with quasi-periodic, finite-gap Schrödinger operators which arise naturally as the stationary solutions of the KdV hierarchy. Hence we only briefly recall some notation and refer to the monograph [13] (see also [28]) for further information.

Let

$$
H_{q}=-\frac{d^{2}}{d x^{2}}+V_{q}(x)
$$

be a finite-gap Schrödinger operator in $L^{2}(\mathbb{R})$ whose spectrum consists of $g+1$ bands:

$$
\sigma\left(H_{q}\right)=\bigcup_{j=0}^{g-1}\left[E_{2 j}, E_{2 j+1}\right] \cup\left[E_{2 g}, \infty\right) .
$$

It is well-known that $H_{q}$ is associated with the Riemann surface $\mathbb{M}$ of the function

$$
R_{2 g+1}^{1 / 2}(z), \quad R_{2 g+1}(z)=\prod_{j=0}^{2 g}\left(z-E_{j}\right), \quad E_{0}<E_{1}<\cdots<E_{2 g},
$$

$g \in \mathbb{N}_{0} . \mathbb{M}$ is a compact, hyperelliptic Riemann surface of genus $g$. Here $R_{2 g+1}^{1 / 2}(z)$ is chosen to have branch cuts along the spectrum with the sign fixed by the asymptotic behavior $R_{2 g+1}^{1 / 2}(z)=\sqrt{z} z^{g}+\ldots$ as $z \rightarrow \infty$.

A point on $\mathbb{M}$ is denoted by $p=\left(z, \pm R_{2 g+1}^{1 / 2}(z)\right)=(z, \pm), z \in \mathbb{C}$. The point at infinity is denoted by $p_{\infty}=(\infty, \infty)$. We use $\pi(p)=z$ for the projection onto the 
extended complex plane $\mathbb{C} \cup\{\infty\}$. The points $\left\{\left(E_{j}, 0\right), 0 \leq j \leq 2 g\right\} \cup\left\{p_{\infty}\right\} \subseteq \mathbb{M}$ are called branch points and the sets

$$
\Pi_{ \pm}=\left\{\left(z, \pm R_{2 g+1}^{1 / 2}(z)\right) \mid z \in \mathbb{C} \backslash \Sigma\right\} \subset \mathbb{M}, \quad \Sigma=\sigma\left(H_{q}\right),
$$

are called upper and lower sheet, respectively. Note that the boundary of $\Pi_{ \pm}$ consists of two copies of $\Sigma$ corresponding to the two limits from the upper and lower half plane.

We recall that upon fixing the spectrum $\sigma\left(H_{q}\right)$, the operator $H_{q}$ is uniquely defined by choosing a Dirichlet divisor

$$
\left\{\left(\mu_{1}, \sigma_{1}\right), \ldots,\left(\mu_{g}, \sigma_{g}\right)\right\}, \quad \mu_{j} \in\left[E_{2 j-1}, E_{2 j}\right] .
$$

For every $z \in \mathbb{C}$ the Baker-Akhiezer functions $\psi_{q, \pm}(z, x)$ are two (weak) solutions of $H_{q} \psi=z \psi$. They are the two branches of one function which is meromorphic on $\mathbb{M} \backslash\left\{p_{\infty}\right\}$ with simple poles at the Dirichlet divisor (2.5) and simple zeros at some other points

$$
\left\{\left(\mu_{1}(x), \sigma_{1}(x)\right), \ldots,\left(\mu_{g}(x), \sigma_{g}(x)\right)\right\}, \quad \mu_{j}(x) \in\left[E_{2 j-1}, E_{2 j}\right],
$$

which can be computed from the Dubrovin equations

$$
\mu_{j}^{\prime}(x)=\frac{-2 \sigma_{j}(x) R_{2 g+1}^{1 / 2}\left(\mu_{j}(x)\right)}{\prod_{k \neq j} \mu_{j}(x)-\mu_{k}(x)}
$$

using the initial conditions $\mu_{j}(0)=\mu_{j}, 1 \leq j \leq g$. Moreover, $V_{q}(x)$ is explicitly given by the trace formula

$$
V_{q}(x)=E_{0}+\sum_{j=1}^{g}\left(E_{2 j-1}+E_{2 j}-2 \mu_{j}(x)\right) .
$$

The Baker-Akhiezer functions are linearly independent away from the band-edges $\left\{E_{j}\right\}_{j=0}^{2 g}$ since their Wronskian is given by

$$
W\left(\psi_{q,-}(z), \psi_{q,+}(z)\right)=\frac{2 \mathrm{i} R_{2 g+1}^{1 / 2}(z)}{\prod_{j=1}^{g}\left(z-\mu_{j}\right)} .
$$

Here $W_{x}(f, g)=f(x) g^{\prime}(x)-f^{\prime}(x) g(x)$ denotes the usual Wronskian and $\mu_{j}$ are the Dirichlet eigenvalues at base point $x_{0}=0$. We recall that $\psi_{q, \pm}(z, x)$ have the form

$$
\psi_{q, \pm}(z, x)=\theta_{q, \pm}(z, x) \exp ( \pm \mathrm{i} x k(z)),
$$

where $\theta_{q, \pm}(z, x)$ is quasi-periodic with respect to $x$ and

$$
k(z)=-\int_{E_{0}}^{p} \omega_{p_{\infty}, 0}, \quad p=(z,+),
$$

denotes the quasimomentum map. Here $\omega_{p_{\infty}, k}$ is a normalized Abelian differential of the second kind with a single pole at $p_{\infty}=(\infty, \infty)$ and principal part $\zeta^{-k-2} d \zeta$ 
where $\zeta=z^{-1 / 2}$. It is explicitly given by

$$
\omega_{p_{\infty}, 0}=-\frac{\prod_{j=1}^{g}\left(\pi-\lambda_{j}\right) d \pi}{2 R_{2 g+1}^{1 / 2}},
$$

where $\lambda_{j} \in\left(E_{2 j-1}, E_{2 j}\right), 1 \leq j \leq g$. In particular, $\left|\mathrm{e}^{\mathrm{i} k(z)}\right|<1$ for $z \in \mathbb{C} \backslash \sigma\left(H_{q}\right)$ and $\left|\mathrm{e}^{\mathrm{i} k(z)}\right|=1$ for $z \in \sigma\left(H_{q}\right)$.

\section{Asymptotics of Jost solutions}

After we have these preparations out of our way, we come to the study of shortrange perturbations $H$ of $H_{q}$ associated with a potential $V$ satisfying $V(x) \rightarrow V_{q}(x)$ as $|x| \rightarrow \infty$. More precisely, we will make the following assumption throughout this paper:

Let

$$
H=-\frac{d^{2}}{d x^{2}}+V(x)
$$

be a perturbation of $H_{q}$ such that

$$
\int_{-\infty}^{+\infty}\left|V(x)-V_{q}(x)\right| d x<\infty
$$

We first establish existence of Jost solutions, that is, solutions of the perturbed operator which asymptotically look like the Baker-Akhiezer solutions.

Theorem 3.1. Assume (3.2). For every $z \in \mathbb{C} \backslash\left\{E_{j}\right\}_{j=0}^{2 g}$ there exist (weak) solutions $\psi_{ \pm}(z,$.$) of H \psi=z \psi$ satisfying

$$
\lim _{x \rightarrow \pm \infty} \mathrm{e}^{\mp \mathrm{i} x k(z)}\left(\psi_{ \pm}(z, x)-\psi_{q, \pm}(z, x)\right)=0
$$

where $\psi_{q, \pm}(z,$.$) are the Baker-Akhiezer functions. Moreover, \psi_{ \pm}(z,$.$) are contin-$ uous (resp. holomorphic) with respect to $z$ whenever $\psi_{q, \pm}(z,$.$) are and$

$$
\left|\mathrm{e}^{\mp \mathrm{i} x k(z)}\left(\psi_{ \pm}(z, x)-\psi_{q, \pm}(z, x)\right)\right| \leq C(z),
$$

where $C(z)$ denotes some constant depending only on $z$.

Proof. Since $H \psi=z \psi$ is equivalent to $\left(H_{q}-z\right) \psi=-\widehat{V} \psi$, where $\widehat{V}=V-V_{q}$, we can use the variation of constants formula to obtain the usual Volterra integral equations for the Jost functions,

$$
\begin{gathered}
\psi_{ \pm}(z, x)=\psi_{q, \pm}(z, x)-\frac{1}{W\left(\psi_{q,+}, \psi_{q,-}\right)} \int_{x}^{ \pm \infty}\left(\psi_{q,-}(z, x) \psi_{q,+}(z, y)-\right. \\
\left.-\psi_{q,-}(z, y) \psi_{q,+}(z, x)\right) \widehat{V}(y) \psi_{ \pm}(z, y) d y .
\end{gathered}
$$

Moreover, introducing $\tilde{\psi}_{ \pm}(z, x)=\mathrm{e}^{\mp \mathrm{i} x k(z)} \psi_{ \pm}(z, x)$ the resulting integral equation can be solved using the method of successive iterations in the usual way. This proves the claims. 
Theorem 3.2. Assume (3.2). The Jost functions have the following asymptotic behavior

$$
\psi_{ \pm}(z, x)=\psi_{q, \pm}(z, x)\left(1 \mp \frac{1}{2 \mathrm{i} \sqrt{z}} \int_{x}^{ \pm \infty}\left(V(y)-V_{q}(y)\right) d y+o\left(z^{-1 / 2}\right)\right),
$$

as $z \rightarrow \infty$, with the error being uniformly in $x$.

Proof. Invoking (3.5) we have

$$
\begin{aligned}
\frac{\psi_{ \pm}(z, x)}{\psi_{q, \pm}(z, x)}=1 & -\frac{1}{W\left(\psi_{q,+}, \psi_{q,-}\right)} \int_{x}^{ \pm \infty}\left(\psi_{q,-}(z, x) \psi_{q,+}(z, y) \frac{\psi_{q, \pm}(z, y)}{\psi_{q, \pm}(z, x)}\right. \\
& \left.-\psi_{q,-}(z, y) \psi_{q,+}(z, x) \frac{\psi_{q, \pm}(z, y)}{\psi_{q, \pm}(z, x)}\right) \widehat{V}(y) \frac{\psi_{ \pm}(z, y)}{\psi_{q, \pm}(z, y)} d y \\
(3.7) \quad & 1 \mp \int_{x}^{ \pm \infty}\left(G_{q}(z, x, x) \frac{\psi_{q, \pm}(z, y)^{2}}{\psi_{q, \pm}(z, x)^{2}}-G_{q}(z, y, y)\right) \widehat{V}(y) \frac{\psi_{ \pm}(z, y)}{\psi_{q, \pm}(z, y)} d y
\end{aligned}
$$

where

$$
G_{q}(z, x, y)=\frac{1}{W\left(\psi_{q,+}, \psi_{q,-}\right)} \begin{cases}\psi_{q,+}(z, x) \psi_{q,-}(z, y), & x \geq y \\ \psi_{q,+}(z, y) \psi_{q,-}(z, x), & x \leq y\end{cases}
$$

is the Green function of $H_{q}$. We have

$$
G_{q}(z, x, x)=\frac{\psi_{q,+}(z, x) \psi_{q,-}(z, x)}{W\left(\psi_{q,+}, \psi_{q,-}\right)}=\frac{\mathrm{i} \prod_{j=1}^{g}\left(z-\mu_{j}(x)\right)}{2 R_{2 g+1}^{1 / 2}(z)} .
$$

Hence for $z$ near $\infty$ one infers

$$
G_{q}(z, x, x)=\frac{\mathrm{i}}{2 \sqrt{z}}\left(1+\frac{1}{2} V_{q}(x) \frac{1}{z}+O\left(\frac{1}{z^{2}}\right)\right),
$$

where we made use of the trace formula (2.8). Next we insert (3.10) into (3.7) such that iteration implies

$$
\frac{\psi_{ \pm}(z, x)}{\psi_{q, \pm}(z, x)}=1 \mp \frac{\mathrm{i}}{2 \sqrt{z}}\left(\int_{x}^{ \pm \infty} \frac{\psi_{q, \pm}(z, y)^{2}}{\psi_{q, \pm}(z, x)^{2}} \widehat{V}(y) d y-\int_{x}^{ \pm \infty} \widehat{V}(y) d y\right)+O\left(\frac{1}{z}\right) .
$$

Next we will show that the first integral vanishes as $\sqrt{z} \rightarrow \infty$. We begin with the case $\operatorname{Im}(\sqrt{z}) \rightarrow \infty$. For that purpose note that

$$
k(z)=\sqrt{z}+c+O\left(z^{-1 / 2}\right), \quad \text { as } z \rightarrow \infty,
$$

for some constant $c \in \mathbb{C}$. Thus we compute

$$
\begin{aligned}
& \left|\int_{x}^{ \pm \infty} \frac{\psi_{q, \pm}(z, y)^{2}}{\psi_{q, \pm}(z, x)^{2}} \widehat{V}(y) d y\right| \leq C \int_{x}^{ \pm \infty} \exp (\mp 2 \operatorname{Im}(\sqrt{z})(y-x))|\widehat{V}(y)| d y \\
& \quad \leq C \int_{x}^{x+\varepsilon}|\widehat{V}(y)| d y+C \cdot \exp (\mp 2 \operatorname{Im}(\sqrt{z}) \varepsilon) \int_{x+\varepsilon}^{ \pm \infty}|\widehat{V}(y)| d y,
\end{aligned}
$$

such that the first integral can be made arbitrary small if $\varepsilon>0$ is small and the second integral vanishes as $\operatorname{Im}(\sqrt{z}) \rightarrow \infty$. 
Otherwise, if $\operatorname{Re}(\sqrt{z}) \rightarrow \infty$, we use (2.10) to rewrite the integral as

$$
\int_{x}^{ \pm \infty}\left(\frac{\theta_{q, \pm}(z, y)^{2}}{\theta_{q, \pm}(z, x)^{2}} \widehat{V}(y) \exp (\mp 2 \operatorname{Im}(\sqrt{z})(y-x))\right) \exp ( \pm 2 \operatorname{iRe}(\sqrt{z})(y-x)) d y
$$

Since

$$
\left|\frac{\theta_{q, \pm}(z, y)^{2}}{\theta_{q, \pm}(z, x)^{2}} \widehat{V}(y) \exp (\mp 2 \operatorname{Im}(\sqrt{z})(y-x))\right| \leq C|\widehat{V}(y)|
$$

the integral vanishes as $\operatorname{Re}(\sqrt{z}) \rightarrow \infty$ by a slight variation of the RiemannLebesgue lemma.

Hence we finally have

$$
\frac{\psi_{ \pm}(z, x)}{\psi_{q, \pm}(z, x)}=1 \pm \frac{\mathrm{i}}{2 \sqrt{z}} \int_{x}^{ \pm \infty} \widehat{V}(y) d y+o\left(\frac{1}{\sqrt{z}}\right)
$$

as $z \rightarrow \infty$.

For later use we note the following immediate consequence

Corollary 3.3. Under the assumptions of the previous theorem we have

$$
\lim _{x \rightarrow \pm \infty} \mathrm{e}^{\mp \mathrm{i} x k(z)}\left(\dot{\psi}_{ \pm}(z, x) \mp \mathrm{i} x \dot{k}(z) \psi_{ \pm}(z, x)-\dot{\psi}_{q, \pm}(z, x) \pm \mathrm{i} x \dot{k}(z) \psi_{q, \pm}(z, x)\right)=0,
$$

where the dot denotes differentiation with respect to $z$.

Proof. Just differentiate (3.3) with respect to $z$, which is permissible by uniform convergence on compact subsets of $\mathbb{C} \backslash\left\{E_{j}\right\}_{j=0}^{2 g}$.

We remark that if we require our perturbation to satisfy the usual short-range assumption as in [1], 9, 10, 11] (i.e., the first moment is integrable, see (5.11), then we even have $\mathrm{e}^{\mp \mathrm{i} x k(z)}\left(\dot{\psi}_{ \pm}(z, x)-\dot{\psi}_{q, \pm}(z, x)\right) \rightarrow 0$.

From Theorem 3.2 we obtain a complete characterization of the spectrum of $H$.

Theorem 3.4. Assume (3.2). Then $(H-z)^{-1}-\left(H_{q}-z\right)^{-1}$ is trace class. In particular, we have $\sigma_{\text {ess }}(H)=\sigma\left(H_{q}\right)$ and the point spectrum of $H$ is confined to $\overline{\mathbb{R} \backslash \sigma\left(H_{q}\right)}$. Furthermore, the essential spectrum of $H$ is purely absolutely continuous except for possible eigenvalues at the band edges.

Proof. That $(H-z)^{-1}-\left(H_{q}-z\right)^{-1}$ is trace class follows as in [37, Lem. 9.34] (cf. also [25, Sect. 4]). The fact that the essential spectrum is purely absolutely continuous follows from subordinacy theory ([37, Sect. 9.5]) since the asymptotics of the Jost solutions imply that no solution is subordinate inside the essential spectrum.

Note that (3.2) does neither exclude eigenvalues at the boundary of the essential spectrum nor an infinite number of eigenvalues inside essential spectral gaps (see [31, 26] or [1 for conditions excluding these cases).

Our next result concerns the asymptotics of the Jost solutions at the other side. 
Lemma 3.5. Assume (3.2). Then the Jost solutions $\psi_{ \pm}(z,),. z \in \mathbb{C} \backslash \sigma(H)$, satisfy

$$
\lim _{x \rightarrow \mp \infty}\left|\mathrm{e}^{\mp \mathrm{i} x k(z)}\left(\psi_{ \pm}(z, x)-\alpha(z) \psi_{q, \pm}(z, x)\right)\right|=0,
$$

where

$$
\alpha(z)=\frac{W\left(\psi_{-}(z), \psi_{+}(z)\right)}{W\left(\psi_{q,-}(z), \psi_{q,+}(z)\right)}=\frac{\prod_{j=1}^{g}\left(z-\mu_{j}\right)}{2 \mathrm{i} R_{2 g+1}^{1 / 2}(z)} W\left(\psi_{-}(z), \psi_{+}(z)\right) .
$$

Proof. Since $H$ and $H_{q}$ have the same form domain, the second resolvent equation (37, Lem. 6.30]) for form perturbations implies

$$
G(z, x, x)-G_{q}(z, x, x)=\int_{-\infty}^{\infty} G(z, x, y) \widehat{V}(y) G_{q}(z, y, x) d y
$$

where $\widehat{V}=V-V_{q}$. By (3.8) and

$$
G(z, x, y)=\frac{1}{W\left(\psi_{+}, \psi_{-}\right)} \begin{cases}\psi_{+}(z, x) \psi_{-}(z, y), & x \geq y \\ \psi_{+}(z, y) \psi_{-}(z, x), & x \leq y\end{cases}
$$

we obtain

$$
\begin{aligned}
G(z, x, x)-G_{q}(z, x, x)= & \frac{\psi_{q,+}(z, x) \psi_{+}(z, x)}{W(z) W_{q}(z)} \int_{-\infty}^{x} \widehat{V}(y) \psi_{q,-}(z, y) \psi_{-}(z, y) d y \\
& +\frac{\psi_{q,-}(z, x) \psi_{-}(z, x)}{W(z) W_{q}(z)} \int_{x}^{\infty} \widehat{V}(y) \psi_{q,+}(z, y) \psi_{+}(z, y) d y,
\end{aligned}
$$

where $W(z)=W\left(\psi_{+}, \psi_{-}\right)$and $W_{q}(z)=W\left(\psi_{q,+}, \psi_{q,-}\right)$. Next, by (3.4), note that

$$
\left|\psi_{q, \pm}(z, x)\right| \leq c_{1} \mathrm{e}^{\mp \varepsilon x}, \quad\left|\psi_{ \pm}(z, x)\right| \leq c_{2} \mathrm{e}^{\mp \varepsilon x},
$$

as $x \rightarrow+\infty$, where $c_{1}, c_{2}$ denote some constants and $\varepsilon>0$ does only depend on $z$. Now one can show that the first term in (3.14) tends to 0 when $x \rightarrow+\infty$ using the same kind of argument as in the proof of Theorem 3.2. Similarly one then checks that the second term in (3.14) tends to 0 when $x \rightarrow-\infty$. Thus

$$
\lim _{x \rightarrow \pm \infty} G(z, x, x)-G_{q}(z, x, x)=0
$$

and using

$$
G_{q}(z, x, x)=\frac{\psi_{q,-}(z, x) \psi_{q,+}(z, x)}{W\left(\psi_{q,-}(z), \psi_{q,+}(z)\right)}, \quad G(z, x, x)=\frac{\psi_{-}(z, x) \psi_{+}(z, x)}{W\left(\psi_{-}(z), \psi_{+}(z)\right)}
$$

implies

$$
\lim _{x \rightarrow \pm \infty}\left(\psi_{-}(z, x) \psi_{+}(z, x)-\alpha(z) \psi_{q,-}(z, x) \psi_{q,+}(z, x)\right)=0,
$$

respectively,

$$
\lim _{x \rightarrow-\infty} \psi_{q,-}(z, x)\left(\psi_{+}(z, x)-\alpha(z) \psi_{q,+}(z, x)\right)=0,
$$

which is the claimed result. 
To see the connection with scattering theory (see, e.g., [1]), we introduce the scattering relations

$$
T(\lambda) \psi_{ \pm}(\lambda, x)=\overline{\psi_{\mp}(\lambda, x)}+R_{\mp}(\lambda) \psi_{\mp}(\lambda, x), \quad \lambda \in \sigma\left(H_{q}\right),
$$

where the transmission and reflection coefficients are defined as usual,

$$
T(\lambda)=\frac{W\left(\overline{\psi_{ \pm}(\lambda)}, \psi_{ \pm}(\lambda)\right)}{W\left(\psi_{\mp}(\lambda), \psi_{ \pm}(\lambda)\right)}, \quad R_{ \pm}(\lambda):=-\frac{W\left(\psi_{\mp}(\lambda), \overline{\psi_{ \pm}(\lambda)}\right)}{W\left(\psi_{\mp}(\lambda), \psi_{ \pm}(\lambda)\right)}, \quad \lambda \in \sigma\left(H_{q}\right) .
$$

In particular, $\alpha(z)$ is just the inverse of the transmission coefficient $T(z)$. It is holomorphic in $\mathbb{C} \backslash \sigma\left(H_{q}\right)$ with simple zeros at the discrete eigenvalues of $H$.

Corollary 3.6. Assume (3.2). Then we have

$$
T(z)=\exp \left(-\int_{-\infty}^{+\infty}\left(m_{ \pm}(z, x)-m_{q, \pm}(z, x)\right) d x\right),
$$

where

$$
m_{ \pm}(z, x)= \pm \frac{\psi_{ \pm}^{\prime}(z, x)}{\psi_{ \pm}(z, x)}, \quad m_{q, \pm}(z, x)= \pm \frac{\psi_{q, \pm}^{\prime}(z, x)}{\psi_{q, \pm}(z, x)}
$$

are the Weyl-Titchmarsh functions. Here the prime denotes differentiation with respect to $x$.

Proof. From the definition (3.19) we get the following representations of the Jost and Baker-Akhiezer functions

$$
\begin{gathered}
\psi_{ \pm}(z, x)=\psi_{ \pm}\left(z, x_{0}\right) \exp \left( \pm \int_{x_{0}}^{x} m_{ \pm}(z, y) d y\right), \\
\psi_{q, \pm}(z, x)=\psi_{q, \pm}\left(z, x_{0}\right) \exp \left( \pm \int_{x_{0}}^{x} m_{q, \pm}(z, y) d y\right),
\end{gathered}
$$

and thus

$$
\begin{aligned}
\frac{\psi_{ \pm}(z, x)}{\psi_{q, \pm}(z, x)} & =\frac{\psi_{ \pm}\left(z, x_{0}\right)}{\psi_{q, \pm}\left(z, x_{0}\right)} \exp \left( \pm \int_{x_{0}}^{x}\left(m_{ \pm}(z, y)-m_{q, \pm}(z, y)\right) d y\right) \\
& =\exp \left( \pm \int_{ \pm \infty}^{x}\left(m_{ \pm}(z, y)-m_{q, \pm}(z, y)\right) d y\right)
\end{aligned}
$$

Making use of that and (3.12) we get

$$
\alpha(z)=\lim _{x \rightarrow \mp \infty} \frac{\psi_{ \pm}(z, x)}{\psi_{q, \pm}(z, x)}=\exp \left( \pm \int_{ \pm \infty}^{\mp \infty}\left(m_{ \pm}(z, y)-m_{q, \pm}(z, y)\right) d y\right),
$$

which finishes the proof.

Corollary 3.7. Assume (3.2). Then T(z) has the following asymptotic behavior

$$
T(z)=1+\frac{1}{2 \mathrm{i} \sqrt{z}} \int_{-\infty}^{\infty}\left(V(y)-V_{q}(y)\right) d y+o\left(z^{-1 / 2}\right),
$$

as $z \rightarrow \infty$.

Proof. Use (3.12) and (3.6). 


\section{Connections with Krein's spectral shift theory and trace formulas}

To establish the connection with Krein's spectral shift theory we next show:

Lemma 4.1. We have

$$
\frac{d}{d z} \alpha(z)=-\alpha(z) \int_{-\infty}^{+\infty}\left(G(z, x, x)-G_{q}(z, x, x)\right) d x, \quad z \in \mathbb{C} \backslash \sigma(H),
$$

where $G(z, x, y)$ and $G_{q}(z, x, y)$ are the Green's functions of $H$ and $H_{q}$, respectively.

Proof. The Lagrange identity ([37, eq. (9.4)) implies

$$
W_{x}\left(\psi_{+}(z), \dot{\psi}_{-}(z)\right)-W_{y}\left(\psi_{+}(z), \dot{\psi}_{-}(z)\right)=\int_{y}^{x} \psi_{+}(z, r) \psi_{-}(z, r) d r,
$$

hence the derivative of the Wronskian can be written as

$$
\begin{aligned}
& \frac{d}{d z} W\left(\psi_{-}(z), \psi_{+}(z)\right)=W_{x}\left(\dot{\psi}_{-}(z), \psi_{+}(z)\right)+W_{x}\left(\psi_{-}(z), \dot{\psi}_{+}(z)\right) \\
& \quad=W_{y}\left(\dot{\psi}_{-}(z), \psi_{+}(z)\right)+W_{x}\left(\psi_{-}(z), \dot{\psi}_{+}(z)\right)-\int_{y}^{x} \psi_{+}(z, r) \psi_{-}(z, r) d r .
\end{aligned}
$$

Using Corollary 3.3 and Lemma 3.5 we have

$$
\begin{aligned}
W_{y}\left(\dot{\psi}_{-}(z), \psi_{+}(z)\right)= & W_{y}\left(\dot{\psi}_{-}+\mathrm{i} \dot{k}_{y} \psi_{-}, \psi_{+}\right)- \\
& \mathrm{i} \dot{k}\left(y W\left(\psi_{-}, \psi_{+}\right)-\psi_{-}(z, y) \psi_{+}(z, y)\right) \\
\rightarrow & \alpha W_{y}\left(\dot{\psi}_{q,-}+\mathrm{i} \dot{k} y \psi_{q,-}, \psi_{q,+}\right)- \\
& \alpha \mathrm{i} \dot{k}\left(y W\left(\psi_{q,-}, \psi_{q,+}\right)-\psi_{q,-}(z, y) \psi_{q,+}(z, y)\right) \\
= & \alpha(z) W_{y}\left(\dot{\psi}_{q,-}(z), \psi_{q,+}(z)\right)
\end{aligned}
$$

as $y \rightarrow-\infty$. Similarly we obtain

$$
W_{x}\left(\psi_{-}(z), \dot{\psi}_{+}(z)\right) \rightarrow \alpha(z) W_{x}\left(\psi_{q,-}(z), \dot{\psi}_{q,+}(z)\right)
$$

as $x \rightarrow+\infty$ and again using (4.2) we have

$$
W_{y}\left(\dot{\psi}_{q,-}(z), \psi_{q,+}(z)\right)=W_{x}\left(\dot{\psi}_{q,-}(z), \psi_{q,+}(z)\right)+\int_{y}^{x} \psi_{q,+}(z, r) \psi_{q,-}(z, r) d r .
$$

Collecting terms we arrive at

$$
\begin{aligned}
\dot{W}\left(\psi_{-}(z), \psi_{+}(z)\right)= & -\int_{-\infty}^{+\infty}\left(\psi_{+}(z, r) \psi_{-}(z, r)-\alpha(z) \psi_{q,+}(z, r) \psi_{q,-}(z, r)\right) d r \\
& +\alpha(z) \dot{W}\left(\psi_{q,-}(z) \psi_{q,+}(z)\right) .
\end{aligned}
$$


Abbreviating $W_{q}=W\left(\psi_{q,-}, \psi_{q,+}\right)$ we now compute

$$
\begin{aligned}
\frac{d}{d z} \alpha(z) & =\frac{d}{d z}\left(\frac{W}{W_{q}}\right)=-\frac{\dot{W}_{q}}{W_{q}^{2}} W+\frac{1}{W_{q}}\left(-\int_{-\infty}^{+\infty}\left(\psi_{+} \psi_{-}-\alpha \psi_{q,+} \psi_{q,-}\right) d r+\alpha \dot{W}_{q}\right) \\
& =-\frac{1}{W_{q}} \int_{-\infty}^{+\infty}\left(\psi_{+}(z, r) \psi_{-}(z, r)-\alpha(z) \psi_{q,+}(z, r) \psi_{q,-}(z, r)\right) d r,
\end{aligned}
$$

which finishes the proof.

Since $(H-z)^{-1}-\left(H_{q}-z\right)^{-1}$ is trace class with continuous integral kernel $G(z, x, x)-$ $G_{q}(z, x, x)$, we have $([2])$

$$
\operatorname{tr}\left((H-z)^{-1}-\left(H_{q}-z\right)^{-1}\right)=\int_{-\infty}^{+\infty}\left(G(z, x, x)-G_{q}(z, x, x)\right) d x, \quad z \in \mathbb{C} \backslash \sigma(H),
$$

and the last result can be rephrased as

$$
\frac{d}{d z} T(z)=T(z) \operatorname{tr}\left((H-z)^{-1}-\left(H_{q}-z\right)^{-1}\right), \quad z \in \mathbb{C} \backslash \sigma(H),
$$

As an immediate consequence we can establish the connection with Krein's spectral shift function ([22]). We refer to 38] for Krein's spectral shift theory in the case when only the resolvent difference is trace class; which is the case needed here.

Theorem 4.2. The transmission coefficient $T(z)$ has the representation

$$
T(z)=\exp \left(\int_{\mathbb{R}} \frac{\xi(\lambda) d \lambda}{\lambda-z}\right)
$$

where

$$
\xi(\lambda)=\frac{1}{\pi} \lim _{\epsilon \downarrow 0} \arg T(\lambda+\mathrm{i} \epsilon)
$$

is the spectral shift function of the pair $H, H_{q}$. Moreover, $\left(V-V_{q}\right)^{1 / 2}\left(H_{q}-z\right)^{-1} \mid V-$ $\left.V_{q}\right|^{1 / 2}$ is trace class and $T(z)$ is the perturbation determinant of the pair $H$ and $H_{q}$ :

$$
T(z)=\operatorname{det}\left(\mathbb{1}+\left(V-V_{q}\right)^{1 / 2}\left(H_{q}-z\right)^{-1}\left|V-V_{q}\right|^{1 / 2}\right) .
$$

If in addition $\left(V-V_{q}\right)\left(H_{q}-z\right)^{-1}$ is trace class we have

$$
T(z)=\operatorname{det}\left(\mathbb{1}+\left(V-V_{q}\right)\left(H_{q}-z\right)^{-1}\right) .
$$

Proof. The function $\operatorname{Im} \log (T(z))$ is a bounded harmonic function in the upper half plane and hence has a Poisson representation (cf. 21])

$$
\operatorname{Im} \log (T(z))=\int_{\mathbb{R}} \frac{y}{(x-\lambda)^{2}+y^{2}} \xi(\lambda) d \lambda . \quad z=x+\mathrm{i} y
$$

Moreover, by $\xi(\lambda)=0$ for $\lambda$ below the spectrum of $H$ and $\xi(\lambda)=O\left(\lambda^{-1 / 2}\right)$ as $\lambda \rightarrow+\infty$ (by Corollary 3.7) we obtain equality in (4.5) up to a real constant. 
The missing constants follows since both sides tend to 1 as $z \rightarrow \infty$. Moreover, combining (4.5) with (4.4) we see

$$
\operatorname{tr}\left((H-z)^{-1}-\left(H_{q}-z\right)^{-1}\right)=\int_{\mathbb{R}} \frac{\xi(\lambda) d \lambda}{(\lambda-z)^{2}},
$$

which shows that $\xi(\lambda)$ is the spectral shift function.

That $T(z)$ is the perturbation determinant is standard if $\left(V-V_{q}\right)\left(H_{q}-z\right)^{-1}$ is trace class (see e.g. 38]) for the slightly more general case when $\left(V-V_{q}\right)^{1 / 2}\left(H_{q}-\right.$ $z)^{-1}\left|V-V_{q}\right|^{1 / 2}$ is trace class we refer to [15, Sect. 4], [18, Sect. 7]. That this last condition holds will be shown in the next lemma below.

To following result needed in the previous proof is of independent interest.

Lemma 4.3. Assume (3.2). Then $\left(V-V_{q}\right)^{1 / 2}\left(H_{q}-z\right)^{-1}\left|V-V_{q}\right|^{1 / 2}$ is trace class. If we even have

$$
\left\|V-V_{q}\right\|_{2 ; 1}=\sum_{n \in \mathbb{Z}}\left(\int_{n}^{n+1}\left|V(x)-V_{q}(x)\right|^{2}\right)^{1 / 2}<\infty,
$$

then $\left(V-V_{q}\right)\left(H_{q}-z\right)^{-1}$ is trace class.

Proof. To see the first claim we begin with the fact [32, Prop. 2.2] that $\mid V-$ $\left.V_{q}\right|^{1 / 2}\left(H_{0}-z\right)^{-1}\left|V-V_{q}\right|^{1 / 2}$ is trace class, where $H_{0}=-\frac{d^{2}}{d x^{2}}$. Let $z<0$ and set $A(z)=\left(V-V_{q}\right)^{1 / 2}\left(H_{0}-z\right)^{-1 / 2}$. Then $A(z) A(z)^{*}=\left|V-V_{q}\right|^{1 / 2}\left(H_{0}-z\right)^{-1}\left|V-V_{q}\right|^{1 / 2}$ is trace class and thus $A(z)$ is Hilbert-Schmidt. In fact, since $A(z)=A\left(z_{0}\right)\left(H_{0}-\right.$ $\left.z_{0}\right)^{1 / 2}\left(H_{0}-z\right)^{-1 / 2}$ this holds for all $z \in \rho\left(H_{0}\right)$ and not just for $z<0$. Hence, using $\left(H_{q}-z\right)^{-1}=\left(H_{0}-z\right)^{-1 / 2} C(z)\left(H_{0}-z\right)^{-1 / 2}$, where $C(z)$ is bounded (cf. [37, Thm. 6.25]), we see $\left|V-V_{q}\right|^{1 / 2}\left(H_{q}-z\right)^{-1}\left|V-V_{q}\right|^{1 / 2}=A C(z) A^{*}$ which establishes the claim.

The see the second claim we again begin with the fact [33, Theorem 4.5] that (4.9) implies that $\left(V-V_{q}\right)\left(H_{0}-z\right)^{-1}$ is trace class. Now the second resolvent equation $\left(H_{q}-z\right)^{-1}=\left(H_{0}-z\right)^{-1}-\left(H_{0}-z\right)^{-1} V_{q}\left(H_{q}-z\right)^{-1}$ establishes the claim since $V_{q}\left(H_{q}-z\right)^{-1}$ is bounded (cf. [37, Sect. 9.7]).

Note that in the case $V_{q}=0$ [33. Prop 4.7] implies that the condition (4.9) is optimal. Moreover, the norm in (4.9) dominates the $L^{1}$ norm, $\|V\|_{1} \leq\|V\|_{2 ; 1}$ by the Cauchy-Schwartz inequality, but the converse is of course not true (since (4.9) forces the function to be locally square integrable).

\section{The transmission coefficient}

Throughout this section we make the somewhat stronger assumption that

$$
\int_{-\infty}^{+\infty}(1+|x|)\left|V(x)-V_{q}(x)\right| d x<\infty
$$


in order to ensure that there is only a finite number of eigenvalues in each gap [31. Our aim is to reconstruct the transmission coefficient $T(z)$ from its boundary values and its poles. To this end, recall that $T(z)$ is meromorphic in $\mathbb{C} \backslash \sigma\left(H_{q}\right)$ with simple poles at the eigenvalues $\rho_{j}$ of $H$. Moreover, for $z \in \sigma\left(H_{q}\right)$ the boundary values from the upper, respectively, lower, half plane exist and satisfy $|T(z)|^{2}=1-\left|R_{ \pm}(z)\right|^{2}$, where $R_{ \pm}(z)$ are the reflection coefficients defined in the previous section.

In the case where $V_{q}=0$, this can be done via the classical Poisson-Jensen formula. In the more general setting here, the reconstruction needs to be done on the underlying Riemann surface. We essentially follow [36, where the analog problem for Jacobi operators was solved.

Denote by $\omega_{p q}$ the normalized Abelian differential of the third kind with poles at $p$ and $q$. Then the Blaschke factor is defined by

$$
B(p, \rho)=\exp (g(p, \rho))=\exp \left(\int_{E_{0}}^{p} \omega_{\rho \rho^{*}}\right)=\exp \left(\int_{E(\rho)}^{\rho} \omega_{p p^{*}}\right), \quad \pi(\rho) \in \mathbb{R},
$$

where $E(\rho)$ is $E_{0}$ if $\rho<E_{0}$ and either $E_{2 j-1}$ or $E_{2 j}$ if $\rho \in\left(E_{2 j-1}, E_{2 j}\right), 1 \leq j \leq g$. It is a multivalued function with a simple zero at $\rho$ and simple pole at $\rho^{*}$ satisfying $|B(p, \rho)|=1, p \in \partial \Pi_{+}$. It is real-valued for $\pi(p) \in\left(-\infty, E_{0}\right)$ and satisfies

$$
B\left(E_{0}, \rho\right)=1 \quad \text { and } B\left(p^{*}, \rho\right)=B\left(p, \rho^{*}\right)=B(p, \rho)^{-1} .
$$

Then we have

Theorem 5.1. The transmission coefficient is given by

$$
T(z, x)=\left(\prod_{j=1}^{g} B\left(p, \rho_{j}\right)^{-1}\right) \exp \left(\frac{1}{2 \pi \mathrm{i}} \int_{\partial \Pi_{+}} \log \left(1-\left|R_{ \pm}\right|^{2}\right) \omega_{p p_{\infty}}\right), \quad p=(z,+),
$$

where we set $R_{ \pm}(p)=R_{ \pm}(z)$ for $p=(z,+)$ and $R_{ \pm}(p)=\overline{R_{ \pm}(z)}$ for $p=(z,-)$.

Proof. Just literally follow the argument in [36, Sect. 3].

Remark 5.2. A few remarks are in order:

(i) Using symmetry, $\left|R_{ \pm}\left(p^{*}\right)\right|=\left|R_{ \pm}(p)\right|$ for $p \in \partial \Pi_{+}$, of the integrand we can rewrite (5.4) as

$T(p, x)=\left(\prod_{j=1}^{g} \exp \left(-\int_{E\left(\rho_{j}\right)}^{\rho_{j}} \omega_{p p^{*}}\right)\right) \exp \left(\frac{1}{2 \pi \mathrm{i}} \int_{\Sigma} \log \left(1-\left|R_{ \pm}\right|^{2}\right) \omega_{p p^{*}}\right)$,

where the integral over $\Sigma$ is taken on the upper sheet.

(ii) There exist explicit formulas for Abelian differentials of the third kind:

$$
\begin{aligned}
\omega_{p q} & =\left(\frac{R_{2 g+1}^{1 / 2}+R_{2 g+1}^{1 / 2}(p)}{2(\pi-\pi(p))}-\frac{R_{2 g+1}^{1 / 2}+R_{2 g+1}^{1 / 2}(q)}{2(\pi-\pi(q))}+P_{p q}(\pi)\right) \frac{d \pi}{R_{2 g+1}^{1 / 2}}, \\
\omega_{p p_{\infty}} & =\left(-\frac{R_{2 g+1}^{1 / 2}+R_{2 g+1}^{1 / 2}(p)}{2(\pi-\pi(p))}+P_{p p_{\infty}}(\pi)\right) \frac{d \pi}{R_{2 g+1}^{1 / 2}},
\end{aligned}
$$


where $P_{p q}(z), P_{p p_{\infty}}(z)$ are polynomials of degree $g-1$ which have to be determined from the normalization condition to have vanishing a-periods. In particular,

$$
\omega_{p p^{*}}=\left(\frac{R_{2 g+1}^{1 / 2}(p)}{\pi-\pi(p)}+P_{p p^{*}}(\pi)\right) \frac{d \pi}{R_{2 g+1}^{1 / 2}} .
$$

(iii) The function

$$
T(p)= \begin{cases}T(z), & p=(z,+), \\ T(z)^{-1}, & p=(z,-),\end{cases}
$$

solves the following scalar meromorphic Riemann-Hilbert factorization problem:

$$
\begin{aligned}
& T_{+}(p, x)=T_{-}(p, x)\left(1-|R(p)|^{2}\right), \quad p \in \partial \Pi_{+}, \\
& (T(p, x))=\mathcal{D}_{\rho}^{*}-\mathcal{D}_{\rho} \\
& T\left(p_{\infty}, x\right)=1
\end{aligned}
$$

Here the subscripts in $T_{ \pm}(p)$ denote the limits from $\Pi_{ \pm}$, respectively. Compare 23], 24].

As was pointed out in [36, this implies the following algebraic constraint on the scattering data.

Theorem 5.3. The transmission coefficient $T$ defined via (5.4) is single-valued if and only if the eigenvalues $\rho_{j}$ and the reflection coefficients $R_{ \pm}$satisfy

$$
\sum_{j} \int_{\rho_{j}^{*}}^{\rho_{j}} \zeta_{\ell}-\frac{1}{2 \pi \mathrm{i}} \int_{\partial \Pi_{+}} \log \left(1-\left|R_{ \pm}\right|^{2}\right) \zeta_{\ell} \in \mathbb{Z} .
$$

\section{Conserved quantities of the KdV hierarchy}

Finally we turn to solutions of the $\mathrm{KdV}$ hierarchy (see [13). Let $V_{q}(x, t)$ be a finite-gap solution of some equation in the $\mathrm{KdV}$ hierarchy, $\operatorname{KdV}_{r}\left(V_{q}(x, t)\right)=0$, and let $V(x, t)$ be another solution, $\operatorname{KdV}_{r}(V(x, t))=0$, such that $V(., t)-V_{q}(., t)$ is Schwartz class for all $t \in \mathbb{R}$. Existence of such solutions has been established only recently in $[8$.

Since the transmission coefficient $T(z, t)=T(z, 0) \equiv T(z)$ is conserved (see 8 - formally this follows from unitary invariance of the determinant), conserved quantities of the $\mathrm{KdV}$ hierarchy can be obtained by computing the asymptotic expansion at $\infty$.

To this end, we begin by recalling the following well-known asymptotics for the Weyl $m$-functions in case of smooth potentials: 
Lemma 6.1. Suppose $V(x) \in C^{\infty}(\mathbb{R})$ is smooth. The Weyl m-functions have the following asymptotic expansion for large $z$

$$
m_{ \pm}(z, x) \asymp \mathrm{i} \sqrt{z} \pm \sum_{n=1}^{\infty} \frac{\chi_{n}(x)}{( \pm 2 \mathrm{i} \sqrt{z})^{n}}
$$

with coefficients defined recursively via

$$
\chi_{1}(x)=V(x), \quad \chi_{n+1}(x)=-\frac{\partial}{\partial x} \chi_{n}(x)-\sum_{m=1}^{n-1} \chi_{n-m}(x) \chi_{m}(x) .
$$

The corresponding expansion coefficients associated with $V_{q}$ will be denoted by $\chi_{q, m}(x)$. It is also known that the even coefficients are complete differentials [4] and the first few are explicitly given by

$$
\begin{aligned}
& \chi_{1}(x)=V(x), \\
& \chi_{2}(x)=-V^{\prime}(x), \\
& \chi_{3}(x)=V^{\prime \prime}(x)-V(x)^{2}, \\
& \chi_{4}(x)=-V^{\prime \prime \prime}(x)+4 V(x) V^{\prime}(x), \\
& \chi_{5}(x)=V^{\prime \prime \prime \prime}(x)-6 V^{\prime \prime}(x) V(x)-5 V^{\prime}(x)^{2}+2 V(x)^{3} .
\end{aligned}
$$

Theorem 6.2. Suppose $V(x)-V_{q}(x) \in \mathcal{S}(\mathbb{R})$ is Schwartz. Then $\log T(z)$ has an asymptotic expansion around $z=\infty$ :

$$
\log T(z) \asymp \mathrm{i} \sqrt{z} \sum_{k=1}^{\infty} \frac{\tau_{k}}{z^{k}} .
$$

The quantities $\tau_{k}$ are given by

$$
\tau_{k}=\int_{-\infty}^{\infty} \frac{\chi_{2 k-1}(x)-\chi_{q, 2 k-1}(x)}{(-1)^{k} 2^{2 k-1}} d x
$$

and are conserved quantities for the KdV hierarchy. Explicitly,

$$
\begin{aligned}
& \tau_{1}=-\frac{1}{2} \int_{-\infty}^{\infty}\left(V(x)-V_{q}(x)\right) d x, \\
& \tau_{2}=-\frac{1}{8} \int_{-\infty}^{\infty}\left(V^{2}(x)-V_{q}^{2}(x)\right) d x, \\
& \tau_{3}=-\frac{1}{32} \int_{-\infty}^{\infty}\left(2 V^{3}(x)-5 V_{x}^{2}(x)-6 V_{x x}(x) V(x)\right. \\
&\left.\quad-2 V_{q}^{3}(x)+5 V_{q, x}^{2}(x)+6 V_{q, x x}(x) V_{q}(x)\right) d x,
\end{aligned}
$$

etc.

Proof. Represent the Jost solutions in the form

$$
\psi_{ \pm}(z, x)=\psi_{q, \pm}(z, x) \exp \left(\mp \int_{x}^{ \pm \infty}\left(m_{ \pm}(z, y)-m_{q, \pm}(z, y)\right) d y\right) .
$$


Then iterating the Volterra integral equations (3.7) one sees that $\psi_{ \pm}(z, x)$ have an asymptotic expansion uniformly with respect to $x$ and given by

$$
\log \frac{\psi_{ \pm}(z, x)}{\psi_{q, \pm}(z, x)} \asymp-\sum_{n=1}^{\infty} \frac{1}{( \pm 2 \mathrm{i} \sqrt{z})^{n}} \int_{x}^{ \pm \infty}\left(\chi_{n}(y)-\chi_{q, n}(y)\right) d y .
$$

Then, letting $x \rightarrow \mp \infty$ using (3.12) yields (6.3). In particular, equality of the plus and minus cases shows that all even expansion coefficients must vanish (which alternatively also follows from the fact that the even expansion coefficients are complete differentials).

Theorem 6.3. Consider the expansion coefficients $\tau_{k}$ of $\log T(z)$ defined in (6.3). Then the following trace formulas are valid:

$$
\tau_{k}=2 \mathrm{i} \sum_{j=1}^{g} \int_{E\left(\rho_{j}\right)}^{\rho_{j}} \omega_{p_{\infty}, 2 k-2}-\frac{1}{\pi} \int_{\Sigma} \log |T|^{2} \omega_{p_{\infty}, 2 k-2},
$$

where $\omega_{p_{\infty}, k}$ is the Abelian differential of the second kind with a pole of order $k+2$ at $p_{\infty}$.

Proof. From $\frac{d^{k}}{d z^{k}} \omega_{p E_{0}}=k ! \omega_{p_{\infty}, k-1}$ we get that

$$
\begin{array}{rlrl}
\omega_{p E_{0}} & =\omega_{p_{\infty} E_{0}}+\sum_{k=1}^{\infty} \zeta^{k} \omega_{p_{\infty}, k-1}, & \zeta=z^{-1 / 2}, \\
\omega_{p^{*} E_{0}}=\omega_{p_{\infty} E_{0}}+\sum_{k=1}^{\infty} \zeta^{k} \omega_{p_{\infty}, k-1}, & \zeta=-z^{-1 / 2} .
\end{array}
$$

Using this it follows

$$
\omega_{p p^{*}}=\omega_{p E_{0}}-\omega_{p^{*} E_{0}}=2 \sum_{k=1}^{\infty} \omega_{p_{\infty}, 2 k-2} \zeta^{2 k-1}, \quad \zeta=z^{-1 / 2} .
$$

Hence we have

$$
\begin{aligned}
& -\sum_{j=1}^{g} \int_{E\left(\rho_{j}\right)}^{\rho_{j}} \omega_{p p^{*}}+\frac{1}{2 \pi \mathrm{i}} \int_{\Sigma} \log |T|^{2} \omega_{p p^{*}}= \\
& -\sum_{j=1}^{g} \int_{E\left(\rho_{j}\right)}^{\rho_{j}} 2 \sum_{k=1}^{\infty} \zeta^{2 k-1} \omega_{p_{\infty}, 2 k-2}+\frac{1}{\pi \mathrm{i}} \int_{\Sigma} \log |T|^{2} \sum_{k=1}^{\infty} \zeta^{2 k-1} \omega_{p_{\infty}, 2 k-2}
\end{aligned}
$$

Thus, since $|T|^{2}=1-\left|R_{ \pm}\right|^{2}$ and $R_{ \pm}(\lambda)$ decays faster than any polynomial as $\lambda \rightarrow \infty$ [8], one obtains

$$
\log T(z) \asymp-\sum_{k=1}^{\infty}\left(2 \sum_{j=1}^{\infty} \int_{E\left(\rho_{j}\right)}^{\rho_{j}} \omega_{p_{\infty}, 2 k-2}-\frac{1}{\pi \mathrm{i}} \int_{\Sigma} \log |T|^{2} \omega_{p_{\infty}, 2 k-2}\right) \zeta^{2 k-1},
$$

where $\zeta=z^{-1 / 2}$ denotes the local coordinate at $z=\infty$. 
Remark 6.4. The differentials $\omega_{p_{\infty}, 2 k-2}, k=1,2, \ldots$, are explicitly given by

$$
\omega_{p_{\infty}, 2 k-2}=\left(\frac{\pi^{g+k-1}}{R_{2 g+1}^{1 / 2}}+P_{k}(\pi)\right) d \pi .
$$

Here $P_{k}(\pi)$ is a polynomial of degree $g+k-2$ which has to be chosen such that all a-periods vanish.

\section{Acknowledgments}

G.T. would like to thank all organizers of the international conference on Operator Theory, Analysis and Mathematical Physics (OTAMP), Bedlewo, June 2008, for their kind invitation and the stimulating atmosphere during the meeting. We are indebted to Iryna Egorova and Fritz Gesztesy for several helpful discussions.

\section{References}

[1] A. Boutet de Monvel, I. Egorova, and G. Teschl, Inverse scattering theory for one-dimensional Schrödinger operators with steplike finite-gap potentials, J. d'Analyse Math. 106:1, 271-316 (2008).

[2] C. Brislawn, Kernels of trace class operators, Proc. Amer. Math Soc. 104:4, 1181-1190 (1988).

[3] P. Deift and R. Killip, On the absolutely continuous spectrum of one-dimensional Schrödinger operators with square summable potentials, Commun. Math. Phys. 203, 341-347 (1999).

[4] P.G. Drazin and R.S. Johnson, Solitons: An Introduction, Cambridge Univ. Press, Cambridge, 1989.

[5] W. Eckhaus and A. Van Harten, The Inverse Scattering Transformation and Solitons: An Introduction, Math. Studies 50, North-Holland, Amsterdam, 1984.

[6] I. Egorova and G. Teschl, On the Cauchy problem for the Korteweg-de Vries equation with steplike finite-gap initial data II. Perturbations with Finite Moments, J. d'Analyse Math. (to appear).

[7] I. Egorova and G. Teschl, A Paley-Wiener theorem for periodic scattering with applications to the Korteweg-de Vries equation, Zh. Mat. Fiz. Anal. Geom. 6:1, 21-33 (2010).

[8] I. Egorova, K. Grunert, and G. Teschl, On the Cauchy problem for the Kortewegde Vries equation with steplike finite-gap initial data I. Schwartz-type perturbations, Nonlinearity 22, 1431-1457 (2009).

[9] N.E. Firsova, An inverse scattering problem for the perturbed Hill operator, Mat. Zametki 18, no. 6, 831-843 (1975).

[10] N.E. Firsova, A direct and inverse scattering problem for a one-dimensional perturbed Hill operator Matem. Sborn. (N.S.) 130(172), no. 3, 349-385 (1986). 
[11] N.E. Firsova, The Riemann surface of a quasimomentum, and scattering theory for a perturbed Hill operator Mathematical questions in the theory of wave propagation, 7. Zap. Naučn. Sem. Leningrad. Otdel. Mat. Inst. Steklov (LOMI) 51, 183-196 (1975).

[12] N. E. Firsova, Solution of the Cauchy problem for the Korteweg-de Vries equation with initial data that are the sum of a periodic and a rapidly decreasing function, Math. USSR-Sb. 63:1, 257-265 (1989).

[13] F. Gesztesy and H. Holden, Soliton Equations and Their Algebro-Geometric Solutions. Volume I: $(1+1)$-Dimensional Continuous Models., Cambridge Studies in Advanced Mathematics, Vol. 79, Cambridge University Press, Cambridge, 2003.

[14] F. Gesztesy and H. Holden, Trace formulas and conservation laws for nonlinear evolution equations, Rev. Math. Phys. 6, 51-95 (1994).

[15] F. Gesztesy and K.A. Makarov, (Modified) Fredholm determinants for operators with matrix-valued semi-separable integral kernels revisited, Integral Eq. Operator Theory 47, 457-497 (2003). (See also Erratum 48, 425-426 (2004) and the corrected electronic only version in 48, 561-602 (2004).)

[16] F. Gesztesy, A. Pushnitski, and B. Simon, On the Koplienko spectral shift function. I. Basics., Zh. Mat. Fiz. Anal. Geom. 4:1, 63-107 (2008).

[17] F. Gesztesy, R. Ratnaseelan, and G. Teschl, The KdV hierarchy and associated trace formulas, in "Proceedings of the International Conference on Applications of Operator Theory", (eds. I. Gohberg, P. Lancaster, and P. N. Shivakumar), Oper. Theory Adv. Appl., 87, Birkhäuser, Basel, 125-163 (1996).

[18] F. Gesztesy, Y. Latushkin, M. Mitrea, and M. Zinchenko, Nonselfadjoint operators, infinite determinants, and some applications, Russ. J. Math. Phys. 12:4, 443-471 (2005).

[19] R. Jost and A. Pais, On the scattering of a particle by a static potential, Phys. Rev. 82, 840-851 (1951).

[20] R. Killip and B. Simon, Sum rules for Jacobi matrices and their applications to spectral theory, Ann. of Math. (2) 158, 253-321 (2003).

[21] P. Koosis, Introduction to $H_{p}$ Spaces, 2nd ed., Cambridge Tracts in Mathematics 115, Cambridge University Press, Cambridge, 1998.

[22] M.G. Krein, Perturbation determinants and a formula for the traces of unitary and self-adjoint operators, Soviet. Math. Dokl. 3, 707-710 (1962).

[23] S. Kamvissis and G. Teschl, Stability of periodic soliton equations under short range perturbations, Phys. Lett. A 364:6, 480-483 (2007).

[24] S. Kamvissis and G. Teschl, Stability of the periodic Toda lattice under short range perturbations, arXiv:0705.0346

[25] H. Krüger and G. Teschl, Relative oscillation theory, zeros of the Wronskian, and the spectral shift function, Comm. Math. Phys. 87:2, 613-640 (2009).

[26] H. Krüger and G. Teschl, Effective Prüfer angles and relative oscillation criteria, J. Diff. Eq. 245, 3823-3848 (2008).

[27] A. Laptev, S. Naboko, and O. Safronov, On new relations between spectral properties of Jacobi matrices and their coefficients, Comm. Math. Phys. 241, no. 1, 91-110 (2003). 
[28] V. A. Marchenko, Sturm-Liouville Operators and Applications, Birkhäuser, Basel, 1986.

[29] J. Michor and G. Teschl, Trace formulas for Jacobi operators in connection with scattering theory for quasi-periodic background, Operator Theory, Analysis, and Mathematical Physics, J. Janas, et al. (eds.), 69-76, Oper. Theory Adv. Appl., 174, Birkhäuser, Basel, 2007.

[30] F. Nazarov, F. Peherstorfer, A. Volberg, and P. Yuditskii, On generalized sum rules for Jacobi matrices, Int. Math. Res. Not. 2005:3, 155-186 (2005).

[31] F.S. Rofe-Beketov, A finiteness test for the number of discrete levels which can be introduced into the gaps of the continuous spectrum by perturbations of a periodic potential, Dokl. Akad. Nauk SSSR 156, 515-518 (1964).

[32] B. Simon, Resonances in one dimension and Fredholm determinants, J. Funct. Anal. 178, 396-420 (2000).

[33] B. Simon, Trace Ideals and Their Applications, 2nd ed., Amer. Math. Soc., Providence, 2005.

[34] B. Simon and A. Zlatoš, Sum rules and the Szegö condition for orthogonal polynomials on the real line, Comm. Math. Phys. 242:3, 393-423 (2003).

[35] G. Teschl, Jacobi Operators and Completely Integrable Nonlinear Lattices, Math. Surv. and Mon. 72, Amer. Math. Soc., Rhode Island, 2000.

[36] G. Teschl, Algebro-geometric constraints on solitons with respect to quasiperiodic backgrounds, Bull. London Math. Soc. 39:4, 677-684 (2007).

[37] G. Teschl, Mathematical Methods in Quantum Mechanics; With Applications to Schrödinger Operators, Graduate Studies in Mathematics 99, Amer. Math. Soc., Providence, 2009.

[38] D.R. Yafaev, Mathematical Scattering Theory: General Theory, Amer. Math. Soc., Rhode Island, 1992.

[39] A. Zlatoš, Sum rules for Jacobi matrices and divergent Lieb-Thirring sums, J. Funct. Anal. 225, no. 2, 371-382 (2005).

Faculty of Mathematics, University of Vienna, Nordbergstrasse 15, 1090 Wien, Austria

E-mail address: Alice.Mikikits-Leitner@univie.ac.at

$U R L:$ http://www.mat.univie.ac.at/ alice/

Faculty of Mathematics, University of Vienna, Nordbergstrasse 15, 1090 Wien, Austria, and International Erwin Schrödinger Institute for Mathematical Physics, Boltzmanngasse 9, 1090 Wien, Austria

E-mail address: Gerald.Teschl@univie.ac.at

$U R L:$ http://www.mat.univie.ac.at/ gerald/ 